\title{
Non-surgical management of spontaneous splenic rupture in infectious mononucleosis
}

\author{
Gerardo Dessì, ${ }^{1}$ Teseo Stefanini, ${ }^{1}$ Giovanni Berisso, ${ }^{2}$ Maja Ruff De Conti, ${ }^{1}$ Alessandra Centi, ${ }^{1}$ Marilisa Carrieri, ${ }^{1}$ \\ Pradeep Sonwalkar ${ }^{3}$
}

${ }^{1}$ Unit of Radiology, Hospital Sant'Andrea, La Spezia, Italy; ${ }^{2}$ Unit of Internal Medicine, Hospital San Bartolomeo, Sarzana (SP), Italy; ${ }^{3}$ BLDEA, S.B.M. Patil Medical College Hospital and Research Center, Bijapur, India

\begin{abstract}
Spontaneous rupture of the spleen is a rare, but serious complication of infectious mononucleosis with no clear consensus on appropriate management. Although management of traumatic splenic rupture has largely moved to non-operative treatment, splenectomy is still frequently used in dealing with rupture of the diseased spleen. Here we report the case of a 27-year-old boy with splenic rupture secondary to laboratory-confirmed infectious mononucleosis in the absence of trauma. Our management included an endovascular treatment (embolization of the splenic artery), serial ultrasound scans, activity limitation, and a computed tomography low-dose scan before discharge. Our experience, along with a review of the literature, has led us to conclude that splenic preservation can be a safe alternative to splenectomy in hemodynamically stable patients with spontaneous splenic rupture. This is of particular importance in the young population, which is at higher risk for postsplenectomy sepsis.
\end{abstract}

\section{Introduction}

Infectious mononucleosis is common among young adults and teenagers, is caused by an infection with the Epstein Barr virus, and has an incidence of $345-671$ cases per 100,000 people per year. ${ }^{1}$ The typical presentation in the pediatric population includes fever, pharyngitis, and lymphadenopathy for 1 to 3 weeks with an acute onset of abdominal pain with signs of hypovolemia. Kehr sign (pain referred to the left shoulder) may also be present in $50 \%$ of patients. ${ }^{2}$ Radiologic confirmation is made with ul-

Correspondence: Gerardo Dessì, via Montefiorino 28, 05100 Terni, Italy.

Mobile: +39.347 .8184143 .

E-mail: gerardo.dessi@gmail.com

Key words: splenic spontaneous rupture, Epstein Barr virus, splenic artery embolization, endovascular treatment, abdominal computed tomography.

Conflict of interests: the authors declare no potential conflict of interests.

Received for publication: 30 November 2013.

Revision received: 9 January 2014.

Accepted for publication: 20 January 2014

This work is licensed under a Creative Commons Attribution NonCommercial 3.0 License (CC BY-NC 3.0).

(C) Copyright G. Dessì et al., 2014

Licensee PAGEPress, Italy

Italian Journal of Medicine 2014; 8:193-195

doi:10.4081/itjm.2014.455 trasound (US) or an abdominal computed tomography (CT) scan.

The majority of patients will have asymptomatic splenomegaly, caused by the proliferation of mononuclear cells in the spleen. However, spontaneous rupture or atraumatic rupture of spleen secondary to infectious mononucleosis is rare, has an estimated incidence of $0.06 \%$ to $0.5 \%{ }^{3}$ and is the most frequent cause of death in infectious mononucleosis.

\section{Case Report}

A previously healthy 27 -year-old boy came to the emergency room with a $24 \mathrm{~h}$ history of epigastric and left hypocondrial pain, fever, malaise, nausea, diarrhea and denied to have a history of trauma. Clinical examination revealed an evidence of localized peritonism on the upper left upper and splenomegaly. Laboratory tests found his hematocrit to be low (27\%). Monospot test results were positive, as were titers for Epstein-Barr virus immunoglobulins $\mathrm{G}$ and $\mathrm{M}$.

Urgent abdominal ultrasound showed splenomegaly and subcapsular hematoma with a significant amount of complex fluid within the abdominal cavity, especially on the left side.

An abdominal CT scan showed splenomegaly (17 $\mathrm{cm}$ in the bipolar diameter) with fractures and hemorragic appearance of the upper pole and active arterial bleeding resulting from a Baltimora grade 4 splenic hematoma with hemoperitoneum (Figure 1). The splenic parenchyma was fractured in multiple planes. A small bilateral pleural effusion was seen.

The patient was immediately referred to the en- 
dovascular unit and submitted in a few minutes to a selective angiography of the celiac trunk with embolization of the proximal side of the splenic artery by the deployment of a $10 \mathrm{~mm}$ Amplazter ${ }^{\mathrm{TM}}$ Plug II (St. Jude Medical, Inc., Saint Paul, MN, USA) (Figure 2).

He was then transferred to the internal medicine

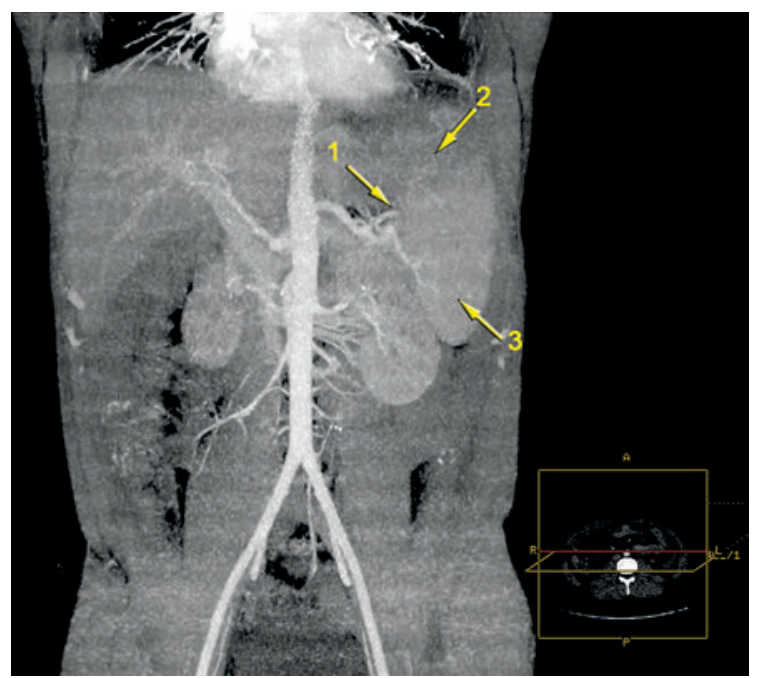

Figure 1. Emergency contrast enhanced computed tomography. Coronal maximum intensive projection reconstruction of arterial phase detects the upper branch of the splenic artery (1) that stops suddenly into the hemorragic splenic parenchyma (2). Normal splenic parenchyma (3) is shown in the lower pole.

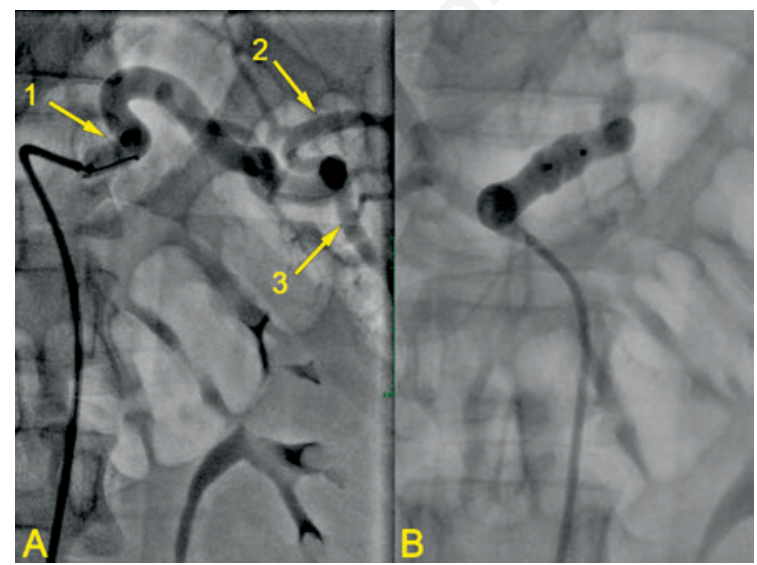

Figure 2. Selective angiography of the celiac trunk (A) with embolization (B) of the proximal side of the splenic artery: deployment of a 10 mm Amplazter ${ }^{\mathrm{TM}}$ Plug II (St. Jude Medical, Inc.) (1) at the origin of the splenic artery, before its bifurcation into the upper (2) and lower (3) branches. At the end of the procedure, the complete embolization of the artery is shown (B). ward: in the following days his hematocrit level returned at 40. A repeat US at four days and CT scan (Figure 3) at ten days showed a decrease of the hemoperitoneum, continued but reduced subcapsular fluid, a decrease of splenomegaly $(15 \mathrm{~cm} \mathrm{vs} 17 \mathrm{~cm})$ and some residual architectural distortion of the upper splenic pole, with no active bleeding signs. A chest radiograph confirmed complete resolution of the pleural effusion.

\section{Discussion}

Despite the fact that infectious mononucleosis is a self-limiting disease, it may cause serious and lethal complications. The mechanism of splenic rupture secondary to infectious mononucleosis has been controversial. It is commonly believed that it is caused by an increase in intra-abdominal pressure or contraction of the diaphragm with vigorous cough, vomiting and defecation, leading to a compression of the spleen.

Histologically, extensive infiltration of the splenic parenchyma with lymphocytes results from fragmentation of the splenic capsule, predisposing to rupture. The spleen is most vulnerable to rupture in the second and third weeks after infection.

In the appropriate clinical context, spleen US can be used to support and differentiate between two of the main splenic complications of infectious mononucleosis, splenic rupture and infarction. Both splenic infarction and rupture are typically managed conservatively, but rupture mandates surgical evaluation for possible

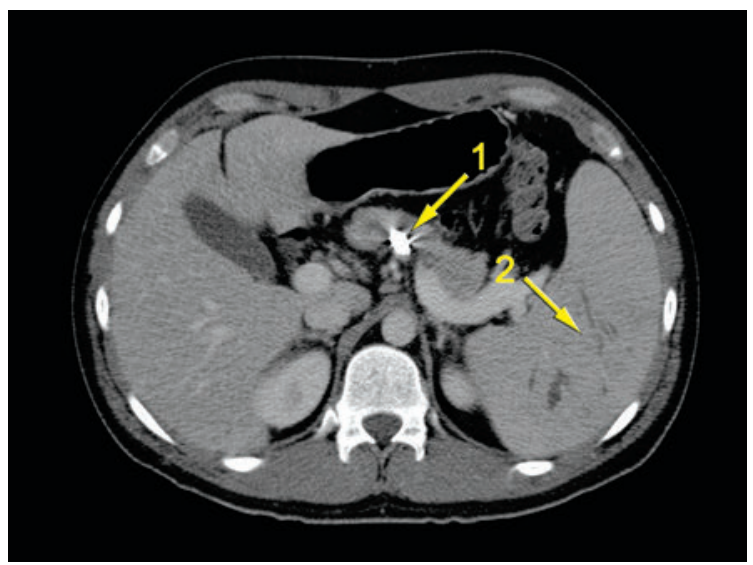

Figure 3. Follow up computed tomography scan at ten days shows the decrease of the hemoperitoneum, continued but reduced subcapsular fluid, the Amplazter ${ }^{\mathrm{TM}}$ Plug II (St. Jude Medical, Inc.) in its side (1), decrease of splenomegaly and some residual architectural distortion of the upper splenic pole (2), with no active bleeding signs. 
splenectomy, and thus, a timely identification of and distinction between these pathologies is essential. ${ }^{4}$

A point-of-care ultrasound for splenic infarct would be expected to show hypoechoic areas in the splenic parenchyma with an absence of internal flow on color Doppler, and no intra-abdominal free fluid. In contrast, as in this case, the sonographic appearance of a splenic rupture shows some sub-capsular or sub-diaphragmatic fluid, which is indicative of blood.

Since the first report of post-splenectomy sepsis by King and Shumacker in 1952, there has been a growing trend toward splenic conservation. ${ }^{5}$ The spleen is vital in protecting against infection by encapsulated organisms, ${ }^{6}$ with an estimated risk of overwhelming sepsis in the asplenic individual at $1 \%$ to $3 \%$, but a mortality rate of $50 \%$ to $80 \%$. Nonoperative management avoids the risk of overwhelming post-splenectomy sepsis, as well as the inherent risks of a laparotomy. Other complications associated with splenectomy include pancreatitis, pneumonia, pulmonary embolism, and a higher rate of wound infections. ${ }^{7}$

The question that still must be answered to ensure that non-operative management is as safe and effective as splenectomy is whether the diseased spleen can heal and retain its immunologic function. Splenomegaly has long been recognized as a complication of infectious mononucleosis (IM) and other infectious conditions and is known to be a self-limited condition. Clinical and scientific studies of the injured healthy spleen have documented that the spleen can retain its full function, while maintaining a low risk for repeat rupture. A growing body of literature is now suggesting that the pathologic spleen can also heal after parenchymal disruption. Guth et al. published a prospective report of 11 patients with splenomegaly that resulted from HIV, IM, leukemia, and sickle-cell disease who experienced isolated splenic rupture, grades 1 to $4 .{ }^{8}$ All 11 patients made a full recovery with $<2$ units of blood transfused per patient. Because of the rarity of spontaneous splenic rupture, there are no current data on immune competence of the spleen after non-operative management, but it is generally assumed that structural healing correlates with continued immune function. ${ }^{7}$

\section{Conclusions}

Our experience, along with a review of the literature, leads us to conclude that splenic preservation made by the endovascular treatment can be a safe alternative to splenectomy in stable patients with spontaneous splenic rupture resulting from infectious mononucleosis. This is especially important to consider in the pediatric and adolescent population, which is at high risk for post-splenectomy sepsis in addition to the risks of non-therapeutic laparotomy.

Although splenectomy has been advocated in the past as the definitive therapy, we recommend transcatheter embolization of the splenic artery. Deterioration of our patient's vital signs, setting of hemodynamic instability, requirement for blood transfusion and expansion of the hematoma seen on US or CT imaging call for splenectomy. ${ }^{9}$

\section{References}

1. Bailey RE. Diagnosis and treatment of infectious mononucleosis. Am Fam Physician 1994;49:879-88.

2. Farley DR, Zietlow SP, Bannon MP, Farnell MB. Spontaneous rupture of the spleen due to infectious mononucleosis. Mayo Clin Proc 1992;67:846-53.

3. Won ACM, Ethell A. Spontaneous splenic rupture resulted from infectious mononucleosis. Int J Surg Case Rep 2012;3:97-9.

4. Mackenzie DC, Liebmann O. Identification of splenic infarction by emergency department ultrasound. J Emerg Med 2013;44:450-2.

5. King H, Shumacker HB Jr. Splenic studies. I. Susceptibility to infection after splenectomy performed in infancy. Ann Surg 1952;136:239-42.

6. Kreuzfelder E, Obertacke U, Erhard J, et al. Alterations of the immune system following splenectomy in childhood. J Trauma 1991;31:358-64.

7. Stephenson JT, DuBois JJ. Nonoperative management of spontaneous splenic rupture in infectious mononucleosis: a case report and review of the literature. Pediatrics 2007; 120:e432-5.

8. Guth AA, Pachter HL, Jacobowitz GR. Rupture of the pathologic spleen: is there a role for nonoperative therapy? J Trauma 1996;41:214-8.

9. Halkic N, Vuilleumier H, Qanadli SD. Spontaneous splenic rupture in infectious mononucleosis treated by embolization of the splenic artery. Can J Surg 2004;47:221-2. 